\title{
Globe
}

Revue internationale d'études québécoises

\section{La jeunesse au Québec. Marges, institutions et représentations}

\section{Lucie Guillemette et Claire Le Brun}

Volume 8, numéro 2, 2005

La jeunesse au Québec. Marges, institutions et représentations

URI : https://id.erudit.org/iderudit/1000907ar

DOI : https://doi.org/10.7202/1000907ar

Aller au sommaire du numéro

Éditeur(s)

Globe, Revue internationale d'études québécoises

ISSN

1481-5869 (imprimé)

1923-8231 (numérique)

Découvrir la revue

Citer ce document

Guillemette, L. \& Le Brun, C. (2005). La jeunesse au Québec. Marges, institutions et représentations. Globe, 8(2), 11-22. https://doi.org/10.7202/1000907ar d'utilisation que vous pouvez consulter en ligne.

https://apropos.erudit.org/fr/usagers/politique-dutilisation/ 


\title{
Introduction
}

\section{La jeunesse au Québec. \\ Marges, institutions et représentations}

\author{
Lucie Guillemette \\ Université du Québec à Trois-Rivières
}

\author{
Claire Le Brun \\ Université Concordia
}

La jeunesse au Québec? Les huit articles réunis dans ce numéro en présentent une vue transversale, multidisciplinaire et diachronique, ce qui nous conduit d'emblée à définir l'objet d'étude. Au sens premier de jeunesse comme âge de la vie et à son emploi métonymique de jeunesse comme groupe de personnes jeunes se sont greffées une multitude d'acceptions. Comme le rappelait Pierre Bourdieu', les rapports entre l'âge social et l'âge biologique sont très complexes. Qu'est-ce qu'être jeune? Qui est jeune? La réponse varie selon l'époque et le lieu, selon les sociétés et même les microsociétés. Elle varie aussi, nous le verrons plus loin, en fonction des champs disciplinaires. Ainsi la définition de la jeunesse n'est-elle pas la même pour les spécialistes de la littérature de jeunesse et pour les psychologues ou les sociologues. En dépit de l'apparition récente de nouvelles catégories éditoriales, telles que "grands adolescents " et " jeunes adultes ", la littérature dite pour la jeunesse vise surtout les enfants et les adolescents, alors que, dans les sciences humaines et sociales, on tend maintenant à réserver le terme " jeunesse " pour la période qui succède à l'adolescence. Si les définitions sont

1. Pierre Bourdieu, " La jeunesse n'est qu'un mot *, Questions de sociologie, Paris, Éditions de Minuit, 1984, p. 144.

Lucie Guillemette et Claire Le Brun, Introduction. La jeunesse au Québec. Marges, institutions et représentations ", Globe. Revue internationale d'études québécoises, vol. 8, n², 2005. 


\section{REVUE INTERNATIONALE D'ÉTUDES QUÉBÉCOISES}

multiples, il reste que la jeunesse constitue un sujet de prédilection dans plusieurs domaines de recherche. Selon Madeleine Gauthier, " on s'intéresse d'abord aux jeunes parce que c'est sur eux que repose l'avenir de la cité $^{2}$ ". Si la jeunesse est un âge de la vie qui varie selon les époques et les sociétés, il n'en demeure pas moins qu'elle "constitue un lieu privilégié d'observation du changement social ${ }^{3}{ }^{n}$.

Comme âge de la vie, la jeunesse précédait traditionnellement l'entrée dans l'âge adulte, marquée par des rites de passage: accès au monde du travail pour les garçons ou, dans certains pays, service militaire, et mariage pour les filles. La durée de cette période intermédiaire pouvait varier selon le milieu social, et la longueur des études et de l'apprentissage. Les sociologues constatent maintenant un retard dans le passage à l'âge adulte. Les étapes, passées autrefois dans un ordre immuable, sont vécues dans le désordre, les jeunes essayant divers styles de vie et changeant plusieurs fois de domicile, d'amis, de conjoint, de vision du monde. Dans les sociétés postindustrielles, la jeunesse semble s'étirer jusqu'à des âges jamais atteints. Le Québec vit comme nombre de pays ce qu'on appelle dans les sociétés francophones le "phénomène Tanguy ^, d'après le film à succès d'Étienne Chatiliez (2001). Le Japon connaît un phénomène identique avec ses * célibataires parasites * qui tardent à fonder une famille en dépit de leurs excellents revenus ${ }^{4}$. De façon générale, en Asie, la tradition du mariage arrangé recule, et les jeunes femmes, qui occupent un emploi, peuvent rester chez leurs parents jusqu'à trente ans.

À quel âge est-on jeune de nos jours? Pour le psychanalyste et psychiatre français Tony Anatrella, l'adolescence se prolongerait jusqu'à la trentaine ${ }^{5}$. Alain Viala considère que les jeunes ont entre seize et vingt-

2. Madeleine GauthiER, - La jeunesse : un mot, mais combien de définitions ? ", Madeleine Gauthier et Jean-François GullLaume [éd.], Définir la jeunesse ? D'un bout à l'autre du monde, Sainte-Foy (Québec), Institut québécois de recherche sur la culture, 1999, p. 12.

3. Madeleine GAUTHIER, Une société sans les jeunes?, Sainte-Foy (Québec), Institut québécois de recherche sur la culture, 1994, p. 17.

4. Paul Orenstein, « Parasites in Prêt-à-porter •, New York Times Magazine, $1^{\mathrm{er}}$ juillet 2001, p. 30-35.

5. Tony Anatrella, Interminables adolescences, les 12-30 ans, Paris, Cerf, 1988. 
cinq ans, tout en soulignant l'inadéquation de cette catégorisation qui amalgame les adultes politiques - l'âge du vote étant dix-huit ans - et les autres ${ }^{6}$. Pour sa part, le psychologue américain Jeffrey Arnett propose d'appeler jeunesse la troisième décennie de la vie, réservant l'appellation a adolescence - pour la seconde ${ }^{7}$. Le gouvernement du Québec rejoint ce découpage dans sa politique de la jeunesse, qui as'adresse principalement au groupe d'âge des 15-29 ans ${ }^{8}$. Si l'on accepte comme définition large de la jeunesse la période de préparation à l'âge adulte, force est de constater qu'elle est de plus en plus longue dans les sociétés riches. Les rites de passage sont facultatifs ou sont vécus à des âges fort différents. L'exemple de la parentalité est l'un des plus éclairants à ce point de vue. En effet, dans les sociétés nord-américaines, coexistent un nombre alarmant de grossesses adolescentes 9 et de grossesses tardives ou sans cesse différées chez les $35-40$ ans. Le cinéma et la télévision québécois ont abondamment représenté ce dernier phénomène en 2005: Maman last call de François Bouvier, d'après un scénario de Nathalie Petrowsky, Horloge biologique de Ricardo Trogi, et, au petit écran, Les Invincibles de Jean-François Rivard et François Létourneau. Les mêmes extrêmes s'observent quant au moment du départ de la maison familiale, des émules de Tanguy aux jeunes de la rue observés dans ce numéro par Marc Perreault.

6. Alain VIALA, "Quelles cultures, et quels jeunes? ", Maryse Souchard, Denis SAINT-JaCQues et Alain VIala [éd.], Les jeunes. Pratiques culturelles et engagement collectif, Québec, Nota bene, 2000, p. 293-298.

7. Jeffrey JENSEN ARNEIT, " Emerging Adulthood. A Theory of Development from the Late Teens through the Twenties ", American Psychologist, vol. 55, $\mathrm{n}^{\circ}$ 5, 2000, p. $469-480$.

8. La jeunesse au cœur du Québec, Secrétariat à la jeunesse, Gouvernement du Québec, 2001, p. 15. Voir aussi p. 16 : * La jeunesse, entendue comme l'ensemble des transitions qui mènent à l'installation dans la vie adulte, tend à s'allonger. Les trajectoires de vie ne sont plus linéaires et se caractérisent de plus en plus par des va-et-vient entre les études, le marché du travail, la vie en appartement seul, en couple ou en colocation - et le domicile familial. .

9. Voir Sylvie HalPern, - Ces enfants qui font des enfants ", Sélection du Reader's Digest, mars 1999, http://www.selection.ca/mag/1999/03/meres.html. Selon cet article, le taux de grossesses adolescentes au Québec aurait augmenté de $56 \%$ en vingt ans. 


\section{REVUE INTERNATIONALE D'ÉTUDES QUÉBÉCOISES}

S'ajoute à cet effet de brouillage des âges la condition de jeunesse revendiquée par une population vieillissante et démographiquement dominante. À cet égard, Robert Hollands établit une distinction utile entre "les cultures de la jeunesse" (celles auxquelles adhèrent les jeunes) et "la culture de jeunesse" où les attributs de la jeunesse sont représentés, reproduits, et auxquels participent même les générations plus âgées $^{10}$ ". Nul besoin de s'étendre sur ce phénomène bien documenté, d'autant que ces rapports à la jeunesse posée comme mode de vie et comme idéal s'avèrent des objets différents. Il y a treize ans paraissait $L a$ génération lyrique de François Ricard ${ }^{11}$. L'essayiste mettait le doigt sur l'impossibilité pour sa génération de concevoir la vieillesse, qu'elle assimilait à l'abdication. Qu'en est-il, en 2005, de cette génération maintenant presque sexagénaire ? Quelle que soit la mise à jour à apporter à l'analyse de Ricard, les données de base ne semblent pas avoir changé. La jeunesse reste la valeur de référence et devient même une obligation morale, sous la nouvelle dictature de la santé et de la beauté ${ }^{12}$. Dans cette foulée, le philosophe français Gilles Lipovetsky définit les * temps hypermodernes " comme une "époque de sensualisation et d'esthétisation de masse des plaisirs ${ }^{13}$ " qui marque pour la jeunesse une nette prédilection.

Comment donc vivre sa jeunesse au sein d'une société éternellement jeune? La question nous mène tout naturellement à aborder la jeunesse comme groupe social. Pour exprimer les choses très simplement, on peut considérer la jeunesse comme le groupe qui se prépare à prendre sa place dans la société des adultes. Le groupe paraîtra d'autant moins menaçant à la société qu'elle est prête à lui donner cette place. Loin d'être une absolue nouveauté, la difficulté d'intégration de la jeunesse a

10. Robert Hollands, - Représenter la jeunesse canadienne. Défis ou possibilité réelle „, Madeleine Gauthier et Diane Picom [éd.], Regards sur... La jeunesse et la sociologie au Canada, Sainte-Foy (Québec), Institut québécois de recherche sur la culture, 2001, p. 111.

11. François RICAKD, La génération lyrique. Essai sur la vie et l'ouvre des premiersnés du baby-boom, Montréal, Boréal, 1992.

12. Lucien SFEZ, La santé parfaite. Critique d'une nouvelle utopie, Paris, Seuil, 1995.

13. Gilles LiPOVETSKY, en coll. avec Sébastien Charles, Les temps hypermodernes, Paris, Grasset, 2004, p. 117. 


\section{MARGES, INSTITUTIONS ET REPRÉSENTATIONS}

de nombreux précédents historiques. Pensons seulement aux analyses de l'historien du Moyen Âge Georges Duby sur la classe chevaleresque au XII ${ }^{e}$ siècle $^{14}$. La jeunesse turbulente est celle des fils impatients d'obtenir un fief, et dont la société aristocratique canalise l'énergie dans des tournois aussi violents que coûteux ou dans des expéditions comme les Croisades, en attendant un mariage et un établissement tardifs. Pour rester dans la même période historique, rappelons que les écoliers qui font peur aux bourgeois dans les villes des xIve et $\mathrm{Xv}^{\mathrm{e}}$ siècles.sont souvent des hors-classe, des diplômés de la faculté des arts qui ne trouvent pas leur voie au sein de l'Église ou dans les grands corps de l'État ${ }^{15}$. C'est ainsi que l'on peut imaginer le jeune Villon se tournant vers les bandes de malfaiteurs. Selon Pierre Bourdieu, jeunesse et vieillesse sont construites socialement dans la lutte entre jeunes et vieux ${ }^{16}$. La représentation de la jeunesse comme turbulente ou violente traduit généralement le malaise social. Dans une étude récente sur la perception de la jeunesse aux ÉtatsUnis, Henry Giroux résume ses propos par la formule "la jeunesse n'est pas à risque, mais le risque ${ }^{17}$. On pourrait aller jusqu'à dire que la jeunesse est souvent une métaphore du changement social perçu et craint. À l'inverse, l'absence de lutte entre les générations n'est pas nécessairement un signe de santé pour une société. Comment oublier qu'au $x^{e}$ siècle les sociétés qui ont accepté et glorifié leur jeunesse ont été les plus totalitaires : jeunesses nazies, communistes, fascistes?

$\mathrm{Au} \mathrm{Xx}$ siècle, la jeunesse est devenue une classe de consommateurs. Aux États-Unis, elle aurait commencé à être perçue comme telle dès les années 1920 , le Jazz $A g e^{18}$. À partir des années 1950 , le phénomène n'a

14. Georges DuBY, Hommes et structures du Moyen Âge, Paris, Flammarion, 1988. 15. Geremek BRONISLAw, Les marginaux parisiens aux xIV et $x v^{e}$ siècles, Paris, Flammarion, 1976.

16. Pierre Bourdieu, op. cit., p. 144.

17. * Rather than being at risk in a society marked by deep economic and social inequalities, youth bave become the risk - (Henry A. GIROux, "The War on the Young: Corporate Culture, Schooling, and the Politics of "Zero Tolerance" *, Ronald StRICKLAND [éd.], Growing Up Postmodern. Neoliberalism and the War on the Young, Lanham, Boulder, New York/Oxford, Rowman/Littlefield Publishers, 2002, p. 35).

18. Bill Osgerby, * A Caste, a Culture, a Market •, Ronald Strickland léd.], op. cit., p. 33 . 
cessé de s'amplifier. Construction idéologique, le teen-ager devient le symbole de changements dans le style de vie américain ${ }^{19}$. Dans cette optique, il est intéressant de rappeler que les anglophones distinguent deux concepts : "adolescence " et - teenagerbood", le premier désignant une période de changements physiologiques et psychologiques, le second référant à une classe socio-économique dans les sociétés industrialisées ${ }^{20}$. Depuis le milieu du $x^{e}$ siècle, donc, toute définition d'une culture de la jeunesse doit prendre en compte le rôle des médias, de la publicité et du commerce dans la définition d'un idéal de jeunesse. Dans quelle mesure les jeunes créent-ils leur propre culture et leur mode d'autoreprésentation? Sont-ils contraints ou libérés par les médias de masse? Les avis diffèrent sur ce point ${ }^{21}$. En effet, certains affirment que les jeunes sont des pantins de la société de consommation :

Volontiers présentés comme des consommateurs acharnés, des êtres superficiels et individualistes, les jeunes d'aujourd'hui n'auraient pour ainsi dire ni boussole, ni sens des responsabilités et des contraintes collectives ${ }^{22}$.

Cependant, d'autres prétendent que "la plupart des spécialistes s'inscrivent en faux contre la thèse de la dépolitisation des jeunes ${ }^{23}$ ", dépolitisation qui serait liée davantage à un rejet de croyances érigées en dogmes qu'à une indifférence et à un individualisme exacerbés. Piot affirme que " [l'implication des jeunes] dans diverses causes prouve qu'ils sont parfaitement capables de s'engager pour défendre leurs idées ${ }^{24}$ ".

19. Joe Austin et Michael Nevin WILLARD, Generations of Youth. Youth Cultures and History in Twentieth-Century America, New York, New York University Press, 1988.

20. Marcel DANESI, Cool. The Signs and Meanings of Adolescence, Toronto, Buffalo et London, University of Toronto Press, 1994.

21. Voir par exemple Pierre BRUNo, Existe-t-il une culture adolescente?, Paris, InPress, 2000 ; Paul Wiuss, Common Culture, Milton Keynes, Open University Press, 1990; et Stephen MiLes, Youth Lifestyles in a Cbanging World, Buckingham, Open University Press, 2000.

22. Olivier PIOT; Adolescents, balte aux clichës !, Toulouse, Milan, 2002, p. 13.

23. Olivier GALland, Sociologie de la jeunesse, Paris, Armand Colin, 2001, p. 197.

24. Olivier PIOT, op. cit., p. 120 . L'auteur se réfère notamment à un épisode faisant état de la mobilisation des jeunes en France : de nombreux étudiants en provenance des lycées ont manifesté à la suite de la désignation de Le Pen comme candidat dans la course à la présidence en avril 2002 . 


\section{MARGES, INSTITUTIONS ET REPRÉSENTATIONS}

Selon un raisonnement qui s'apparente à celui mis de l'avant par Bourdieu, des chercheurs vont jusqu'à clamer que "l'adolescence n'existe pas $^{25}$. et qu'il s'agit d'un artifice, d'une pure invention des sociétés postindustrielles ${ }^{26}$. Nul doute que le propos de Jocelyn Létourneau, qui tente de circonscrire le "lieu identitaire de la jeunesse * est plus dialectique. À une époque où la globalisation des marchés a engendré une culture planétaire, "le défi pour les jeunes était d'inventer les passerelles leur permettant de faire du mondial et du local, du présent et du passé ${ }^{27}$. Tout se passe comme si la jeunesse "se [construisait] justement à la jonction du passé et de l'avenir, des héritages et des changements, plantée au carrefour du connu et de l'inconnu ${ }^{28}$. n

Quelles que soient les représentations et autoreprésentations de la jeunesse, une réalité est devenue incontournable au seuil du $\mathrm{xx}^{\mathrm{e}}$ siècle : la jeunesse est un groupe social extrêmement hétérogène. Avec les déplacements accrus de population, différentes expériences de jeunesse coexistent sur le même territoire : coexistence rarement harmonieuse, comme le rappelle brutalement la récente vague de violence dans les banlieues françaises. Le sociologue Pierre Bruno serait enclin à ne voir qu'un leurre dans la reconnaissance de la jeunesse et dans l'importance attribuée à ce groupe. La * croyance en cette jeunesse " amplifierait selon lui l'inégalité des chances ${ }^{29}$. Il paraît clair en effet que la longue période de maturation qu'est devenue la jeunesse - étape de préparation à l'entrée dans un monde du travail de plus en plus complexe - ne peut que favoriser les classes les plus aisées, les mieux informées du fonctionnement socio-économique, les plus à l'aise dans les technologies de l'information.

25. Pierte Hutrre, Martine Pagan-Raymond et Jean Michel Raymond [éd.], L'adolescence n'existe pas. Histoire des tribulations d'un artifice, Paris, Éditions Universitaires, 1990.

26. Pensons aux enfants de l'époque préindustrielle au Canada, qui entraient très rapidement dans la vie adulte alors qu'ils aidaient leurs parents à la ferme.

27. Jocelyn LÉTOURNEAU, - Présentation •, Le lieu identitaire de la jeunesse d'aujourd'bui. Études de cas, Jocelyn LÉTOURNEAu [éd.], Paris et Montréal, L'Harmattan, 1997, p. 14.

28. Madeleine GAUTHIER, Une sociêté sans les jeunes?, p. 17.

29. Pierre Bruno, * La jeunesse en libération conditionnelle -, Maryse SOUCHARD, Denis Salnt-Jacques et Alain Viala léd.], op. cit., p. 153-163. 
Groupe dominé et en attente d'une autonomie (financière, politique, etc.), la jeunesse est fortement dépendante des institutions, qu'elles se présentent sous forme de l'État, de l'école, de la famille ou, plus tristement pour certains, du centre de détention. À d'autres époques, l'orphelinat et le pensionnat ont également joué un rôle important. L'histoire de l'édition pour la jeunesse révèle aussi le pouvoir considérable assumé par l'institution littéraire. La jeunesse était l'âge où on devait être éduqué, dressé ou redressé ; elle est devenue l'âge où on se forme. Comment l'institution a-t-elle évolué dans les sociétés moins prescriptivés qui vivent un véritable brouillage des rites de passage ? Il est intéressant de rappeler les grandes lignes du document publié par le Secrétariat à la jeunesse du gouvernement du Québec en 2001, La jeunesse au cœur du Québec: l'État entend "engager la société dans une culture de la relève ${ }^{30}$ ", et appelle à un " changement durable des mentalités à l'égard des jeunes et [à] une reconnaissance de leurs valeurs ". Dans les pays postindustrialisés, l'idée commence à se répandre que les jeunes devraient participer plus activement aux changements macro-sociétaux ${ }^{31}$. Le chantier est donc "ouvert ", comme l'écrit Madeleine Gauthier, pour qui "le retour de l'acteur * constitue l'étape la plus récente de l'histoire des représentations de la jeunesse au Québec.

Les thématiques qui sous-tendent l'ensemble des articles présentés ici se greffent à deux axes principaux de recherche : le premier prend en compte les structures historiques, sociales et économiques, et le second s'attache aux représentations et aux productions culturelles. Ainsi, si les textes du présent dossier s'inscrivent dans différents champs disciplinaires, il n'en demeure pas moins qu'ils s'articulent autour d'un pôle de recherche commun, celui de la jeunesse au Québec, au croisement de ces deux axes. Ceux-ci répondent à notre double souci de rendre compte de la dynamique intégrative des articles, en même temps que des problèmes, des enjeux et des pratiques ressortissant au problème plus général de l'articulation entre ces axes.

30. François LegaulT, ministre d'État à l'Éducation et à la Jeunesse, La jeunesse au cceur du Québec, op. cit., p. 9.

31. Voir par exemple Jeylan T. Mortimer et Michael J. Shanahan [éd.], Handbook of the Life Course, New York, Kluwer Academic \& Plenum Publishers, 2003. 
Sur le premier axe se trouvent les articles à visée historique et sociologique qui figurent au dossier, c'est-à-dire les textes de Madeleine Gauthier, Christine Hudon et Louise Bienvenue, Sylvie Ménard et Marc Perreault. D'entrée de jeu, Gauthier aborde la jeunesse au Québec sous l'angle des changements socio-économiques, "qui contribuent à la transformation des rapports d'âge •. L'auteure * [se] propose de diviser en quatre grandes périodes les représentations de la jeunesse qui [lui] semblent définir les rapports entre ceux qu'on nomme "jeunes" et les autres âges de la vie au cours du dernier siècle jusqu'à aujourd'hui. "La démonstration vise à faire de la jeunesse une catégorie autonome et différente des autres âges de la vie.

En situant leur recherche dans l'histoire de l'éducation au Québec, Christine Hudon et Louise Bienvenue mettent en lumière l'importance des dispositifs de contrôle de la jeunesse à partir de l'analyse de la correspondance et des journaux intimes qu'ont laissés maîtres et élèves du collège de Sainte-Anne au tournant du $x x^{e}$ siècle. Par la voie de la micro-histoire (ou de l'histoire au quotidien), il s'agit pour les auteures de comprendre la conception de la jeunesse qui anime l'œuvre éducative, si l'on pense à l'analyse qu'elles produisent des écrits de Wilfrid Lebon, enseignant au collège de Sainte-Anne.

Sylvie Ménard examine pour sa part " la place qu'occupe l'institutionnalisation dans les stratégies de contrôle de la délinquance juvénile au Québec entre les années 1869 et 1950. "Tandis que la gestion des écoles de réforme et des écoles d'industrie est confiée à des communautés religieuses à l'époque de la Confédération, la situation se transforme au tournant du xxe siècle, au moment où sont ouverts en Amérique du Nord des tribunaux pour mineurs permettant l'implantation de mesures en milieu ouvert. Comme l'explique Ménard, l'Institut Saint-Antoine reste mal adapté au nouveau système de justice des mineurs institué par l'ouverture de la Cour des jeunes délinquants de Montréal en 1912.

La délinquance juvénile associée aux bandes de jeunes constitue l'objet d'investigation de Marc Perreault, qui s'attache au phénomène urbain lié à la présence des jeunes dans les rues. Au terme de l'enquête qu'il a menée auprès des jeunes en milieu urbain, l'auteur tente de 
distinguer les diverses formes d'appartenance à des bandes et souligne la confusion entourant le statut des jeunes arpentant les rues - qu'ils fassent partie d'un gang ou non. En décrivant les gangs de rue et leurs dérives dans la violence et la criminalité, Perreault préconise "une approche compréhensive et préventive" visant à mieux cerner les stratégies identitaires des jeunes marginaux, et ce, dans le but d'éviter toute forme d'escalade dans la violence.

Les articles de Franck Le Coroller, Lucie Guillemette, Claire Le Brun et Suzanne Pouliot s'inscrivent sur le second axe, celui des représentations et des productions culturelles. Franck Le Coroller, d'abord, cherche à reproduire la trajectoire identitaire de jeunes graffiteurs qu'il a rencontrés et accompagnés lors de leurs pérégrinations nocturnes, pendant une période donnée. Posé comme une marque identitaire, "le graffiti est un exemple de rituel qui compense l'absence d'épreuves autrefois vécues par les jeunes *. Il s'agit alors de rendre compte de la créativité et de l'agentivité sociale des jeunes mâles qui ont trouvé un moyen de s'autoreprésenter à travers les productions dont ils émaillent divers lieux urbains, notamment les dépôts de trains.

Si l'agentivité des graffiteurs suscite un questionnement d'ordre identitaire, celle des jeunes filles rêvant d'un prince charmant a retenu l'attention de Lucie Guillemette. L'auteure examine trois romans d'Anne Hébert qui ont paru dans les années 1980 et 1990 et qui présentent des adolescentes: Les fous de Bassan (1982), L'enfant chargé de songes (1992) et Aurélien, Clara, Mademoiselle et le Lieutenant anglais (1995). À partir d'un appareil de lecture fondé sur le féminisme, elle décrit et analyse le parcours des jeunes héroïnes romanesques qui, pourtant assujetties à l'idéal amoureux des fictions du patriarcat, énoncent leurs désirs et posent des actions leur faisant écho. Selon cette perspective, Guillemette étudie dans les fictions hébertiennes les figures féminines de l'adolescence, que. leurs productions discursives transforment en de véritables sujets. Elles peuvent ainsi franchir les étapes les projetant sur la voie de l'autodétermination et, par ricochet, de l'agentivité féministe.

Les représentations de l'adolescence sont également au centre des préoccupations de Claire Le Brun, qui se propose de jeter un regard 


\section{MARGES, INSTITUTIONS ET REPRÉSENTATIONS}

d'ensemble sur les sept romans pour adolescents de Raymond Plante, parus entre 1986 et 1998 . Plus précisément, l'auteure se penche sur diverses figures esthétiques (personnages, espace-temps, techniques narratives, intertextualité) qui ponctuent cette cuvre pour la jeunesse. À la lumière de ces résultats, Le Brun montre que les fictions romanesques de l'écrivain présentent des traits esthétiques caractéristiques du romantisme français, de Chateaubriand à George Sand. Comme l'indique l'auteure à propos des personnages de Plante, "la conscience aiguë de leur marginalité est en profonde affinité avec l'éthos romantique . Contrairement à de nombreux romans pour adolescents publiés durant cette période et qui témoignent du nombrilisme des jeunes personnages, "un discours valorisant le respect de l'autre et la tendresse "traverse les romans de cet écrivain.

C'est au monde de l'édition pour la jeunesse que s'intéresse pour sa part Suzanne Pouliot dans un article où huit décennies sont revues et étudiées. Alors qu'elle reconstitue l'histoire de l'édition pour un jeune lectorat, l'auteure circonscrit une image de la jeunesse telle qu'elle apparaît dans les diverses collections destinées à un jeune public tout au long du $\mathrm{xx}^{e}$ siècle. De cette réflexion se dégagent des considérations ayant trait à l'histoire du livre et de la lecture au siècle dernier. Pouliot démontre en fait que " les transformations sociales qui ont eu lieu depuis les années 1960 se retrouvent à divers degrés dans la production éditoriale romanesque et albumique ", faisant ainsi de l'édition pour la jeunesse " un lieu privilégié d'observation du changement social ", pour reprendre les termes de Madeleine Gauthier.

Les articles rassemblés dans ce dossier consacré à la jeunesse au Québec font état de problématiques multiples se déployant sur la trajectoire de deux axes de recherche, l'un dit structurel, l'autre défini comme culturel : représentations et autoreprésentations d'adolescents et d'adolescentes en quête d'agentivité ou de romantisme; délinquance juvénile, institutionnalisation et gangs de rue ; édition pour la jeunesse et histoire du livre ; mission éducative et conception de la jeunesse ; autonomie de la jeunesse en tant qu'âge de la vie. Chacun à leur manière, les textes qui suivent exposent la diversité des questionnements épistémologiques et théoriques suscités par l'objet d'étude retenu. Bien que plusieurs 


\section{REVUE INTERNATIONALE D'ÉTUDES QUÉBÉCOISES}

éléments n'aient point été abordés et que beaucoup reste à dire sur le sujet, il demeure que les textes qui composent ce dossier montrent, à divers degrés, que "la jeunesse, c'est ce passage durant lequel vont se construire presque définitivement, alors qu'elles sont encore en pointillé, les coordonnées sociales de l'individu 32 ..

32. Olivier GaLlaND, op. cit., p. 63. 